\title{
The True Catalyst revealed: The Intervention of Chiral $\mathrm{Ca}$ and $\mathrm{Mg}$ Phosphates in Brønsted Acid Promoted Asymmetric Mannich Reac- tions.
}

\author{
Luis Simón ${ }^{a^{*}}$ and Robert S. Paton ${ }^{\mathrm{b} *}$ \\ ${ }^{a}$ Facultad de Ciencias Químicas, Universidad de Salamanca, Plaza de los caídos 1-5, Salamanca 37008, Spain. \\ ${ }^{b}$ Chemistry Research Laboratory, University of Oxford, 12 Mansfield Road, Oxford OX1 3TA, UK \\ KEYWORDS. Metal Combo Catalyst, Chiral Phosphoric Acids, Ca(II) and Mg(II) catalyst, Asymmetric Catalysis, \\ Reaction Mechanism.
}

\begin{abstract}
The acetylacetone-benzaldimine Mannich reaction catalysed by $\mathrm{Mg}(\mathrm{II})$ and $\mathrm{Ca}$ (II) salts of chiral phosphoric acids (CPA) has been investigated computationally by QM/MM methods. Enantioselectivity in this reaction is both larger, and in the opposite sense to that observed for the same reaction catalysed by the protic CPA catalyst alone. We present a mechanistic model from which the characteristic differences between these metal and metal-free catalysts, which can co-exist in the same reaction mixture, can be understood. Alkaline earth salts with chiral phosphate counterions are found to be more catalytically active than the protic form, and the $\mathrm{Ca}$ (II) and $\mathrm{Mg}$ (II) CPA salts react via different mechanisms, with a higher coordination number favored by calcium over magnesium. In the well-ordered chiral cavities around these metal centers, asymmetric induction arises from the steric interaction with the imine protecting group in the unfavourable pathway, with both substrates adopting well-defined conformations. These mechanistic models have allowed us to rationalize the stereochemical outcome across a range of bimolecular reactions promoted by divalent metal phosphates formed with different CPAs.
\end{abstract}

\section{INTRODUCTION}

Asymmetric organocatalysis has been enhanced greatly by the introduction of chiral phosphoric acids (CPAs) by Akiyama and Terada. ${ }^{1-10}$ Recent attention has focussed on combining these catalysts with a range of metals to explore new modes of activation. Asymmetric examples of this have been reported using metal salts formed from $\mathrm{Ag}(\mathrm{I}),{ }^{11-18} \mathrm{Mg}(\mathrm{II}),{ }^{19-22} \mathrm{Ca}(\mathrm{II}),{ }^{23-30} \mathrm{Au}(\mathrm{I}),{ }^{31} \mathrm{Mn}(\mathrm{III}),{ }^{32} \mathrm{Fe}(\mathrm{III}),{ }^{33}$ $\mathrm{Cu}(\mathrm{I})^{34}$ etc. $^{7,35-38}$ and CPA counteranions. Stereochemical models for these transformations are still in their infancy. In contrast, the mechanistic details of Brønsted acid organocatalysis with CPAs have received considerable theoretical ${ }^{39-52}$ and experimental $\left.\right|^{53-56}$ attention. Stereochemical models predict the degree and sense of the selectivity observed depending on the nature of the reagents $52,57,58$ and on the size of the CPA 3,3'substituents..$^{59-61}$ Metal-phosphate catalysts have also been studied, ${ }^{15,21,24,51,62-72}$ but a general model for the selectivity is more difficult to formulate. Multiple substrate binding modes may occur, distinct from the well-defined bifunctional interaction made by the CPA in its protonated form, which are computationally challenging and must be assessed. ${ }^{72}$

Ishihara has studied the reaction of benzaldimine $\mathbf{1}$ and acetylacetone 2 promoted by a CPA, and also by its salts with $\mathrm{Ca}^{2+}$ and $\mathrm{Mg}^{2+}$ cations (Figure 1). ${ }^{73}$ Using an $\mathrm{HCl}$ washed CPA catalyst $(\mathbf{4 a} \cdot \mathbf{H}$ or $\mathbf{4 b} \cdot \mathbf{H})$, the enantioselectivity was moderate, whereas $\mathrm{Mg}^{2+}$ or $\mathrm{Ca}^{2+}$ salts ([4a $]_{2} \mathbf{M g}$ and $[4 \mathbf{a}]_{2} \mathrm{Ca}$, gave greater enantioselectivity, Although the absolute configuration of the CPA remained unchanged in these experiments, stereoinduction due to metal vs. protic CPA catalysts was found to be in the opposite sense. Most strikingly, using a CPA purified by column chromatography in the absence of added $\mathrm{Mg}^{2+}$ or $\mathrm{Ca}^{2+}$ salts, the sense and degree of enantioselectivity matched the $[4 \mathbf{a}]_{2} \mathrm{Ca}$ salt. Exchange of the acidic proton with the metal cation over silica gel is the likely cause. These important results highlight that CPAmetal salts can form inadvertently during CPA purification and may in fact intervene in Brønsted acid catalysed reactions. Occasionally, the CPA salt will be an inactive impurity, requiring higher catalyst loading. More importantly, metal phosphates may also be catalytically active, and either enhance or erode the enantioselectivity due to the CPA organocatalyst alone. The mechanism of catalysis by $[4 a]_{2} \mathbf{M g}$ and $[4 a]_{2} \mathrm{Ca}$, and the basis for the reversal in selectivity observed relative to the protic form of the catalyst have yet to be addressed.
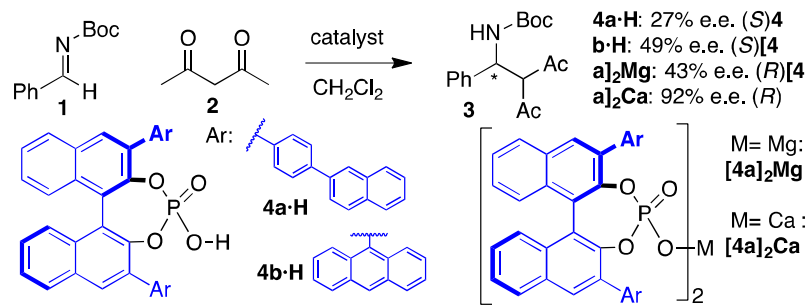
Figure 1. Stereoselective addition of acetylacetone to NBoc benzaldehyde imine is promoted by chiral phosphoric acids and by their $\mathrm{Ca}^{2+}$ or $\mathrm{Mg}^{2+}$ salts with characteristically different enantioselectivities.

Ishihara proposed bifunctional (Lewis acid/general base) activation in the reaction of $\mathbf{1}$ and $\mathbf{2}$ promoted by $[4 a]_{2} \mathbf{C a}$. In this model, the imine is activated by the nitrogen atom binding to a $\mathrm{Ca}(\mathrm{II})$ center and the enol nucleophile forms an $\mathrm{H}$-bond to a phosphate oxygen (Figure 2a). ${ }^{73}$ Antilla envisaged a similar activation mode in the Aza-Darzens reaction catalysed by (VAPOL) ${ }_{2} \mathrm{Mg}$, albeit one in which the benzoyl imine binds to the metal center at $\mathrm{O}$ rather than $\mathrm{N}$ (Figure $2 \mathrm{~b}$ ). ${ }^{20}$ Chlorination of oxindoles by NCS catalysed by (VAPOL) ${ }_{2} \mathrm{Ca}$ was anticipated to behave similarly (Figure 2c). ${ }^{25}$ Antilla $^{26}$ proposed a model for the $(\mathrm{CPA})_{2} \mathrm{Ca}$ catalysed hetero-DA cycloaddition of dienes and 1,2 dicarbonyl compounds (Figure 2d). In contrast to previous models, a monodentate binding mode of each CPA was proposed based on the catalyst $\mathrm{X}$-ray structure. Masson ${ }^{24}$ observed a nonlinear effect across scalemic calcium phosphate catalyst compositions in addition of enamides to azadicarboxylates, implicating the formation of homo- and heterochiral complexes with more than one CPA group (Figure $2 \mathrm{e})$. NMR experiments confirmed the existence of higher order oligomers in solution. The participation of (CPA) ${ }_{2} \mathrm{M}$ complexes is also suggested in other work. ${ }^{23,27,28}$

a)

$$
\text { a) }
$$

Figure 2. Mechanistic proposals for reactions promoted by divalent metal phosphate $\mathrm{M}(\mathrm{CPA})_{2}$ organocatalysts.

The participation of 1:1 $\mathrm{CPA}^{-\mathrm{M}^{2+}}$ complexes has also been suggested. Luo described the combination of a CPA organocatalyst with $\mathrm{MgCl}_{2}$ in the intramolecular tertaminocyclization of 2-arylidenemalonates. ${ }^{21}$ The stereodetermining step was proposed to involve a 1:1 CPA$\mathrm{Mg}^{2+}$ complex which binds to the enolate at the metal center (Figure 2f). Similarly, a 1:1 CPA-In(III)Br complex was proposed in the hetero-DA reaction of cyclopentadienes and $\beta, \gamma$-unsaturated- $\alpha$-ketoesters (Figure $2 g$ ). List proposed a CPA-Pd(II)(allyl) complex in the $\alpha$-allylation of enamines (Figure $2 \mathrm{~h})^{74}$ which was investigated computationally by Sunoj. ${ }^{62}$ In addition, Sunoj has also investigated 2:1 complexes in other CPA-Pd(II) catalyzed reactions. ${ }^{63,64}$

We have computationally studied the reaction of acetylacetone 2 and imine $\mathbf{1}$ catalysed by chiral $\mathrm{Mg}$ (II) and $\mathrm{Ca}$ (II) $4 a$ salts. We have evaluated the stability and the reactivity of the catalytic species formed with one or two CPA counteranions. These mechanisms and their enantioselectivities are compared with those obtained for the metal-free, protic forms of $\mathbf{4 a} \cdot \mathbf{H}$ and $\mathbf{4 b} \cdot \mathbf{H}$. New stereochemical models have been derived to explain the qualitative differences in stereoselectivity obtained using $\mathrm{Ca} / \mathrm{Mg}$ and protic CPA catalysts.

\section{APPROACH}

Automatic procedures for exploring the conformational space of catalysts and reagents based on Monte Carlo sampling and triage by the use of semi-empirical or MM methods have been described:75,76 in this case we were able to evaluate conformations systematically and exhaustively based on chemical reasoning. M06-2X/6$311+\mathrm{G}(\mathrm{d}, \mathrm{p}) / / \mathrm{B} 3 \mathrm{~L} Y \mathrm{Y} / 6-31 \mathrm{G}(\mathrm{d}, \mathrm{p})$ relative energies were used to compare reactant conformations and configurations.

(a) electrophile: acyl-imine $\mathbf{1}$ can adopt a $Z$ or $E$ conformation with respect to the single $\mathrm{C}-\mathrm{N}$ bond (Figure $3 \mathrm{a}$ ). The $Z$ conformer is only $1.4 \mathrm{kcal} / \mathrm{mol}$ less stable (the difference is similar for the amine), so both are plausible.

(b) nucleophile: acetylacetone 2 enol can adopt Z- or Eand $s$-cis or s-trans configurations (Figure $3 \mathrm{~b}$ ). The most stable of these is Z-s-cis. ${ }^{77,78}$ An intramolecular $\mathrm{H}$-bond stabilizes this conformation by $>10 \mathrm{kcal} / \mathrm{mol}$ over the others. The enolate of $\mathbf{2}$ (cf. the neutral enol) lacks an intramolecular $\mathrm{H}$-bond and the most stable configuration is $E$-s-cis (for the anion this is equivalent to Z-s-trans). The preference for the $E$-enolate was found previously in guanidinium catalysis. ${ }^{79}$ We explored all possible conformations of 2 in each TS.

(c) catalyst: $\beta$-naphthtyl 3,3'-substituents of catalyst $\mathbf{4 a} \cdot \mathbf{H}$ can each adopt two orientations. In a TS with one CPA, 4 conformers are possible; for two CPAs this increases combinatorially to $2^{4}=16$. Exploring all these possibilities requires considerable computational effort. We explored these conformational degrees of freedom only for those TSs within $4 \mathrm{kcal} / \mathrm{mol}$ (for TSs involving $1 \mathrm{CPA}$ molecule) or $9 \mathrm{kcal} / \mathrm{mol}$ (for TSs involving $2 \mathrm{CPA}$ molecules) of the most stable TSs. Only the most stable TSs are presented throughout, although the full Boltzmann ensemble of structures was used to compute enantioselectivities. Where 4 (or 16) conformations were calculated, their energy spanned ca. $2 \mathrm{kcal} / \mathrm{mol}$ (1 CPA molecule) or ca. $3 \mathrm{kcal} / \mathrm{mol}$ (2 CPA molecules). This justifies the threshold of $4 \mathrm{kcal} / \mathrm{mol}$ or $9 \mathrm{kcal} / \mathrm{mol}$ described above.

(d) absolute configuration: attack of either imine enantioface is possible (Figure $3 \mathrm{~d}$ ).

(e) binding mode: TSs in which 2 is $\mathrm{H}$-bonded to the catalyst (e.g. in reactions catalysed by $\mathbf{4 a} \cdot \mathbf{H}$ or $\mathbf{4 b} \cdot \mathbf{H}$ ) the $\mathbf{H}$ bond between phosphate and enol oxygen atoms can be either $s$-cis or $s$-trans with respect to the $\mathrm{C}=\mathrm{C}$ bond (Figure 3d). 

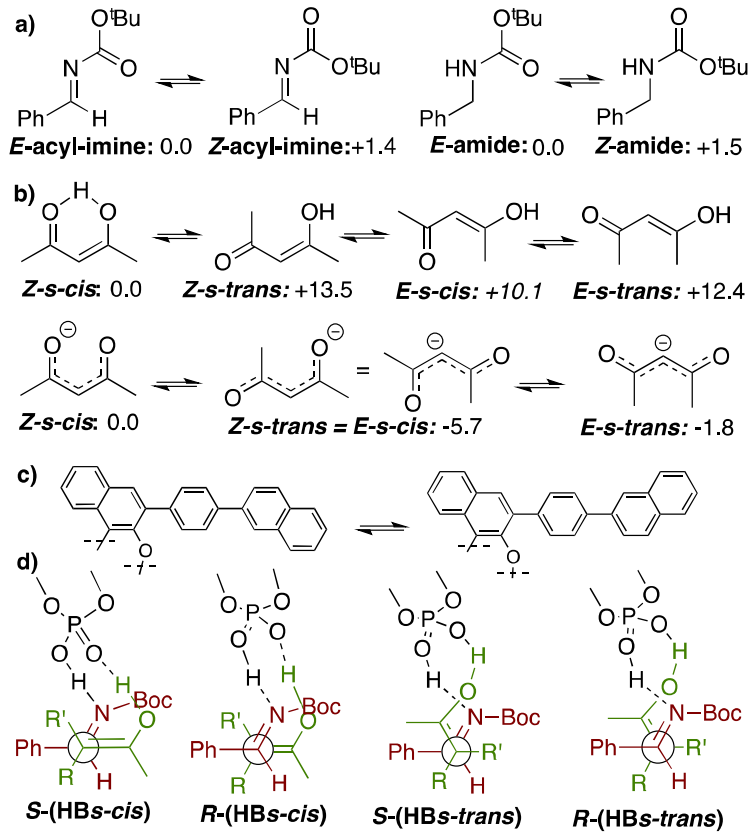

Figure 3. Enumeration of possible conformations for the reagents, CPA catalyst and binding modes.

Full computational methods can be found in the Supporting Information. These methods consist of ONIOM QM:MM geometry optimizations followed by DFT calculation of the energy on the resulting structures, an approach which has provided reasonable agreement with experimental results in studies of similar systems. $39,42,46,52,57,79-84$ In computing the relative Gibbs energies of competing TSs, we considered the magnitude of possible errors associated with our description of thermal corrections and of long-range electron correlation (i.e. dispersion). A quasi-harmonic description of low-lying vibrational frequencies was used to obtain entropic terms, according to models proposed by Grimme ${ }^{86}$ or Cramer and Truhlar. ${ }^{87}$ TS Gibbs energies differ on average by $0.4 \mathrm{kcal} / \mathrm{mol}$ between the two treatments (Fig S4 and S5), although when used to compute levels of enantioselectivity, we only saw differences $4-13 \%$ e.e. We considered the effect of long-range dispersion, evaluating the M06-2X energies with and without a D3correction. ${ }^{88}$ Due to the large catalyst size, we deemed it necessary to include the repulsive Axilrod-Teller-Muto 3body term. Although we consider it desirable to include the dispersion correction, ${ }^{89-92}$ the absence of a D3-term led only to minor changes with respect to the energy differences, and small changes in the evaluation of ee. Only in one case did this change the ee by more than $5 \%$. It is notable that the UFF method performs rather well in its description of aliphatic and aromatic hydrocarbon nonbonding potentials. For such interactions, this method gives quantitatively comparable results when compared to $\operatorname{CCSD}(\mathrm{T}) / \mathrm{CBS}$ and other dispersion corrected functionals (Figure 4). In our mechanicallyembedded QM/MM calculations, nonbonding interactions between the high and low levels are treated at the UFF level, and thus we expect comparable geometries as full M06-2X or B3LYP-D3 optimization. This same cannot be said for a semi-empirical, HF or DFT low-level partition.

Quantitative calculations of stereoselectivity have been performed according to the statistical assumptions of transition state theory and upon application of the Curtin-Hammett principle: we assume rapid equilibration of diastereomeric pre-reactive complexes relative to $\mathrm{C}-\mathrm{C}$ bond formation. ${ }^{93}$ The computed relative Gibbs energies of competing $\mathrm{C}-\mathrm{C}$ forming TSs are compared. This step is computed to occur irreversibly and is therefore stereodetermining. Enantiomeric excess (ee) values were calculated from a Boltzmann ensemble at 298.15 K. Since the feasibility of each mechanism is established by the comparision of TS energies involving the same number of reagents and catalyst units, our conclusions are not affected by possible problems in the estimation of translational entropy. ${ }^{94-96}$ All energies shown in figures and used in the discussion are referred to the most stable TS structure for each catalyst. Therefore, large energy values correspond to less stable TSs.

In addition to catalysts $\mathbf{4 a}$ and $\mathbf{4 b}$ which were studied experimentally, we also performed calculations for model catalyst $\mathbf{4 h}$, lacking 3,3'-substituents to identify the effect of these substituents.
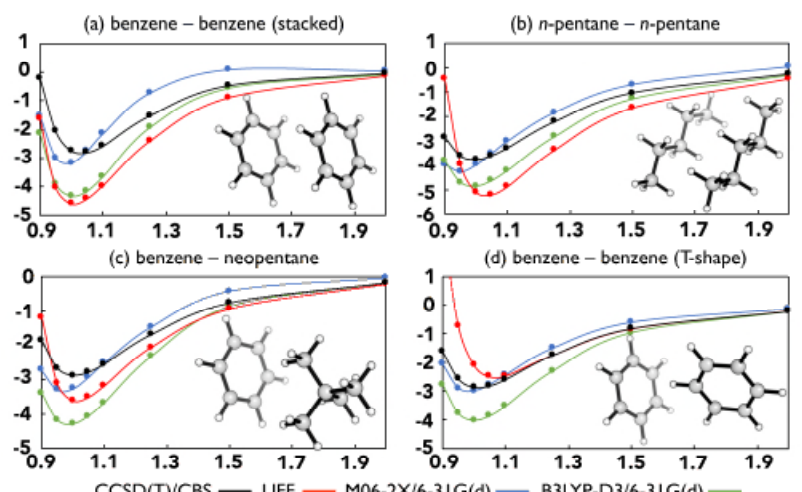

Figure 4. Dissociation curves $(\mathrm{kcal} / \mathrm{mol})$ for four noncovalent complexes representative of catalyst:substrate interactions. A small 6-31G(d) basis set was used for DFT calculations to reflect usage for geometry optimizations.

\section{RESULTS AND DISCUSSION}

Reaction catalysed by $[4 \mathrm{a}]_{2} \mathrm{Mg}$ and $[4 \mathrm{a}]_{2} \mathrm{Ca}$. We considered four mechanistic possibilities (Figure 5). A single CPA ligand is present in mechanism la/lb, whereas two chiral ligands are present in mechanisms Ila/llb; substrate coordination modes differ between the pathways a and $\mathbf{b}$. Mechanism Ila' is similar to Ila but with a higher coordination number of the central atom. Mechanisms la and $\mathrm{Ib}$ can be discarded based on the large energy barrier and the disagreement between calculated and experimental results. The relative energies of the TSs also show mechanism Illb as the most feasible for $[4 \mathrm{a}]_{2} \mathbf{M g}$, while mechanism Ila' is preferred for $[4 a]_{2} \mathbf{C a}$ (see details in SI section). 


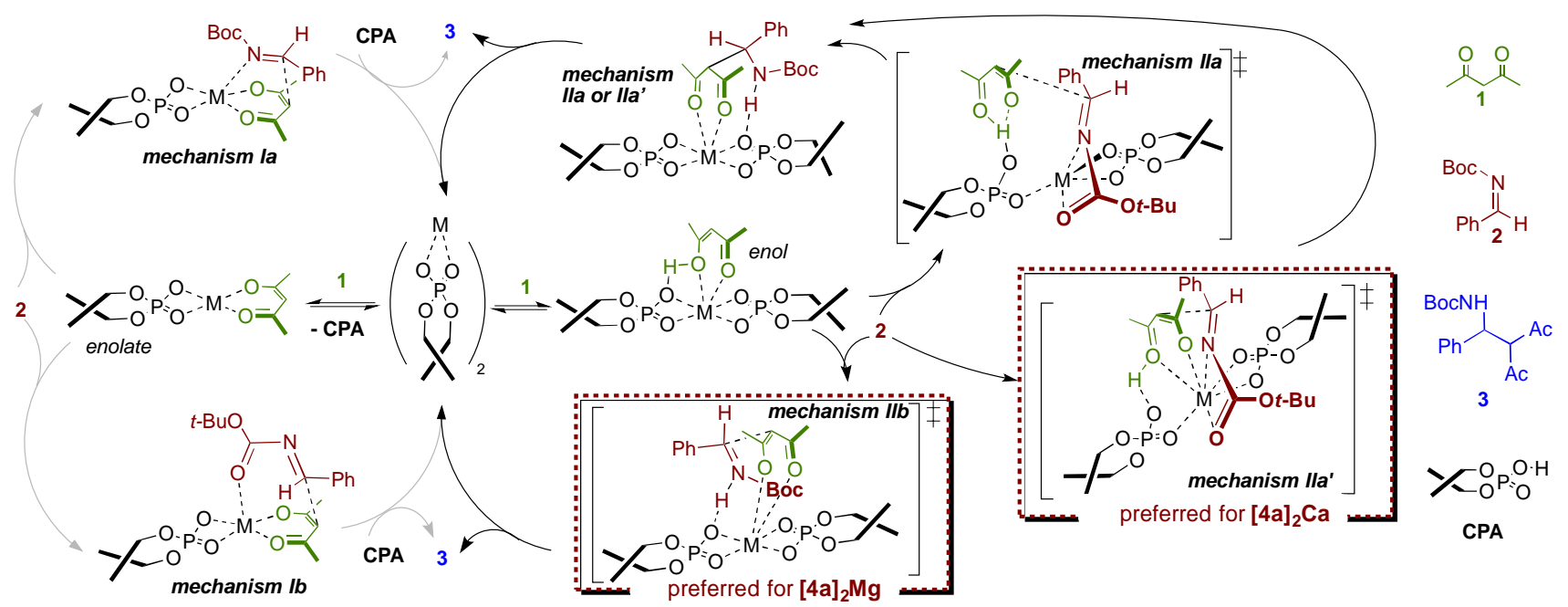

Figure 5. Different mechanisms considered for the reaction catalysed by $[4 \mathbf{a}]_{2} \mathbf{M g}$ and $[\mathbf{4 a}]_{2} \mathbf{C a}$ catalysts.

We also examined three orientations of the two $4 \mathbf{a}$ phosphates (details in supporting information). The most stable TSs correspond to the structure shown in Figure $5 \mathrm{a}$ for the complex of $[\mathbf{4 a}]_{2} \mathbf{M g}$ and the enolate of $\mathbf{2}$. In the front-on perspective, the upper-left and lower right quadrants are occupied by 4-( $\beta$-naphthtyl)-phenyl substituents from both CPA units, as shown in the steric map in the frontal projection.

For the $[4 \mathrm{a}]_{2} \mathbf{M g}$ reaction following mechanism $\mathrm{Ilb}$, the enolate is in all cases in the Z-s-cis conformation, with both oxygen atoms coordinated to the $\mathrm{Mg}^{2+}$ center. Both $E$ or $Z$ conformations of the imine are possible. In the most stable $(R)$-TS, [4a] $]_{2} \mathbf{M g}-\mathbf{I l b}-\mathbf{E}_{\mathbf{i m}-(\mathbf{R})}$ (Figure 6), the $E$ acyl-imine is oriented with the bulky Boc group into an open quadrant of the catalyst. In (S)-TS [4a] $]_{2} \mathbf{M g}-\mathbf{I l b}-\mathbf{E}_{\mathbf{i m}}-$ (S) the positions of Boc and phenyl groups are swapped, which is $4.7 \mathrm{kcal} / \mathrm{mol}$ higher in energy. The $Z$ conformation of the acyl-imine alleviates these steric interac-

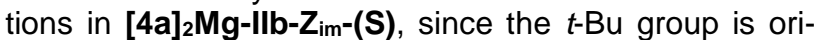
ented away from the catalyst substituents (+0.9 $\mathrm{kcal} / \mathrm{mol}$ ). This TS is the most populated to form the minor enantiomer. This imine conformation is not advantageous for the $(R)$-pathway, with $[\mathbf{4} \mathbf{a}]_{2} \mathbf{M g}-\mathbf{I l b}-\mathbf{Z}_{\mathrm{im}}-(\mathbf{R})$ lying $2.5 \mathrm{kcal} / \mathrm{mol}$ above the most stable TS. The product distribution, calculated from the contribution of all TSs found for mechanisms Ila and IIb is $52 \%-56 \%$ ee in favor of the $R$-product. This agrees with the sense and magnitude of experimental results $(43 \% \mathrm{ee}, R)$.

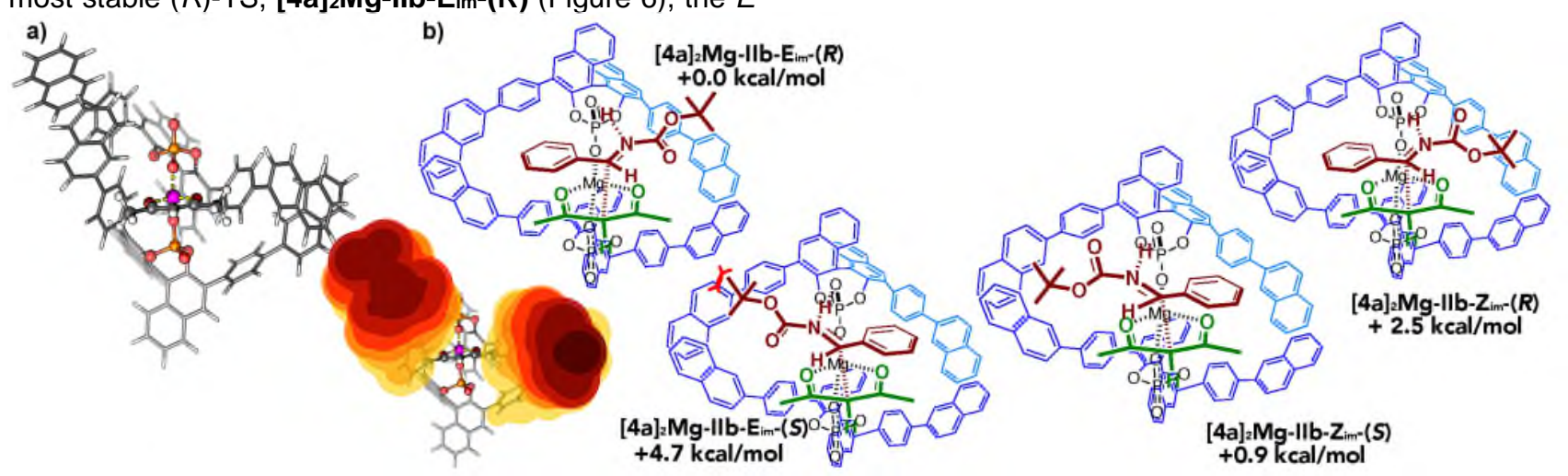

Figure 6. Top: frontal view of the catalyst $[4 \mathrm{a}]_{2} \mathbf{M g}$ complexed with the enolate of $\mathbf{2}$, and steric map of this projection; bottom: Most stable TSs for $[\mathbf{4} \mathbf{a}]_{2} \mathbf{M g}$ proceeding via mechanism Ilb. 3-D representations of these structures are in the Supporting Information section.

For catalyst [4a] $]_{2} \mathbf{C a}$ and mechanism Ila' the enolate conformation is fixed, but there are four possible relative orientations of the reagents. In a frontal view (Figure 7 , top), the enolate can be either to the right (Ila' TSs) or to the left (Ila"' TSs). Newman projection of the forming C-C bond (Figure 7, bottom) also shows two orientations of the imine BOC: toward the protonated (Ila'-(S) and Ila'"$(\boldsymbol{R})$ TSs) or the anionic (Ila"-( $\boldsymbol{R})$ and Ila'-(S) TSs) BINOP unit. TSs with the BOC oriented toward the protonated unit (3.5 for Ila"'-( $\boldsymbol{R})$ and $4.0 \mathrm{kcal} / \mathrm{mol}$ for Ila'-(S) TSs) are more stable for $[\mathbf{4 h}]_{2} \mathrm{Ca}$ catalyst. However, this arrangement also lead the bulky BOC group toward the catalyst, so steric interactions with the side substituents revert this higher stability for $[\mathbf{4 h}]_{2} \mathrm{Ca}$ catalyst. The TSs in which the larger $\mathrm{N}$-acyl-imine is to the left, toward the 4-( $\beta$-naphththyl)-phenyl substituent that blocks the upper-left quadrant (Figure 7 and steric map in Figure 6), are less stable. Accordingly, the most stable TSs corre- 
spond to $[4 \mathrm{a}]_{2} \mathrm{Ca}-\mathrm{Ila}-(\boldsymbol{R})$, in which the BOC is outward and the enolate is to the right side. In the less stable

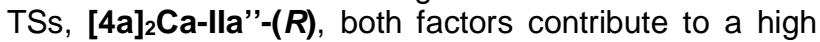
energy, $+3.8 \mathrm{kcal} / \mathrm{mol}$. The relative instability of $[4 \mathrm{a}]_{2} \mathrm{Ca}-$ Ila'-(S) (+1.9 kcal/mol) and [4a] $]_{2}$ Ca-lla'"-(S) (+2.2 $\mathrm{kcal} / \mathrm{mol}$ ) can be attributed, respectively, to the "inward" orientation of the $\mathrm{BOC}$ group and to the placement of the imine reagent to the most hindered left side. The smaller
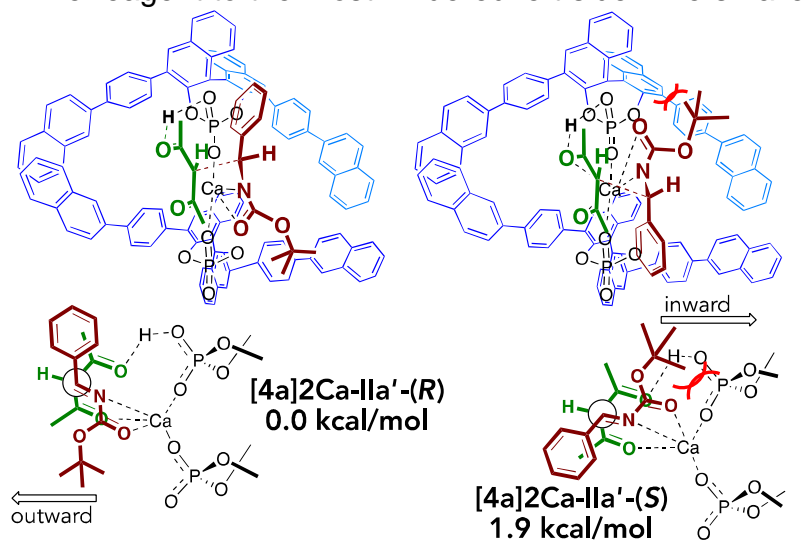

steric effects in lla'-( $\boldsymbol{R})$ TSs is also shown by the small $\operatorname{RMSD}(0.318 \AA)$ between TSs of $[4 \mathbf{a}]_{2} \mathrm{Ca}$ and $[4 \mathbf{h}]_{2} \mathrm{Ca}$ catalyst. The other TSs require larger deformation (RMSD $=0.704,0.663$ and $0.468 \AA$ for lla'-(S), Ila'"-(S) and Ila"'-(R), respectively) to reduce steric interactions. Calculations predict a $92-86 \%$ ee for the $(R)$-product, consistent with experimental results of $95 \%$ ee. ${ }^{97}$

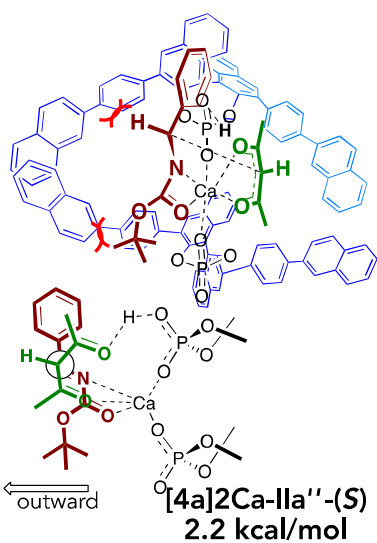

Figure 7: Most stable TSs for [4a] ${ }_{2}$ Ca proceeding via mechanism Ila'. 3-D representations of these structures are in the Supporting Information section.

Distortion-interaction/activation-strain analyses suggest enantioselectivity has different origins for $[4 \mathbf{a}]_{2} \mathbf{M g}$ and $[4 \mathrm{a}]_{2} \mathrm{Ca}$. For $[4 \mathbf{a}]_{2} \mathbf{M g}$, less favorable TSs suffer from worse interaction energies. The lack of catalyst:substrate geometric complementarity in these orientations leads to weaker binding of the iminium to the catalyst. For $[4 a]_{2} \mathbf{C a}$, less favorable TSs suffer from greater catalyst distortion energies. In this case, reorganization of the catalyst/enol complex needs to take place to accommodate the substrate for minor TSs. In the operative mechanism for $[4 \mathrm{a}]_{2} \mathrm{Ca}$ complexes the imine is held firmly in place by dual interactions with the metal at $\mathrm{N}$ and $\mathrm{O}$, so that the catalyst:enol complex is forced to adjust around the imine, causing greater discrimination between major and minor pathways. The fact that steric interactions with the N-Boc substituent are responsible of the chiral assistance by $[4 \mathrm{a}]_{2} \mathrm{Ca}$ is consistent with the results of Gridnev and Terada ${ }^{98}$ for a related catalyst employing different $\mathrm{N}$ substituents, assuming that, as proposed by Ishihara, the reaction is also in this case catalysed by the $\mathrm{Ca}^{2+}$ salt of the catalyst. Asymmetric induction is lower for smaller substrates ( $\mathrm{N}-\mathrm{CO}_{2} \mathrm{Me}$ and $\mathrm{N}-\mathrm{Cbz}$ substituents giving $6 \%$ and $26 \%$ ee, respectively) compared to the $\mathrm{N}$ Boc substrate $(90 \%$ ee), consistent with the key steric influence at this position.

The results for catalysts $[4 \mathrm{a}]_{2} \mathrm{Ca}$ and $[4 \mathrm{~b}]_{2} \mathbf{M g}$ can be used to explain selectivity in other reactions catalysed by $\mathrm{Mg}$ (II) and $\mathrm{Ca}$ (II) CPA salts. The catalyst will, in all cases, adopt an arrangement of the CPA units similar to that in the TS structures shown above (Figures $6 \& 7$ ). For reactions of 1,3 dicarbonyl compounds catalysed by $(\mathrm{CPA})_{2} \mathrm{Mg}$, mechanism Ilb will be preferred: the enolate, fixed in the Z-s-cis conformation, will coordinate the $\mathrm{Mg}^{2+}$ center and the electrophile will be $\mathrm{H}$-bonded to the pro- tonated CPA unit; the preferred orientation will place the largest substituent to the more hindered side of the CPA. Antilla's aza-Darzens reaction between benzoyl imines and 3-chloropentane-2,4-dione catalyzed by (VAPOL) ${ }_{2} \mathrm{Mg}^{2+}, 20$ is described by this model, placing the benzoyl group to the less hindered left side (Figure 8a, note that, in the $((R)-\mathrm{VAPOL})_{2} \mathrm{Mg}^{2+}$ salt, the most hindered catalyst quadrant is top-right and not top-left, and that the benzoyl substituent "extends further" from the reacting center of the imine than the phenyl group). The less stable Z-imine conformation also justifies the larger chiral assistance. A similar argument justifies the enantioselectivity observed for the $((R) \text {-BINOP })_{2} \mathrm{Mg}^{2+}$ catalyzed addition of 2-chloro-ethanol to benzoylimines in Antilla's oxazolidine synthesis ${ }^{22}$ (Figure $8 \mathrm{~b}$ ).

The preference of $(\mathrm{CPA})_{2} \mathrm{Ca}$ salts for larger coordination numbers of the metal atom favour mechanism Ila'. The bulkier reagent will be placed on the less hindered side of the catalyst active center with its bulkier side pointing away of the nearest catalyst flanking substituents (downward, in the catalyst representation in Figure 6). Antilla ${ }^{25,30}$ studied nucleophilic additions to N-Boc-3aryloxindoles catalysed by $((R)-\mathrm{VAPOL})_{2} \mathrm{Ca}^{2+}$ salts. In our model, the electrophile will be placed in the catalyst parallel to the catalyst aromatic sheet in the bulkier topright quadrant, leading the N-Boc downwards to prevent steric interactions. The nucleophile is placed to the less hindered left side (Figure $8 \mathrm{c}$ ). The (BINOP) ${ }_{2} \mathrm{Ca}^{2+}$ catalyzed reaction between azodicarboxylates and enamides, ${ }^{24}$ can be described by this model: the largest diisopropyl-azodicarboxylate will be placed in the less hindered right side, with the less sterically demanding enamide to the left oriented to allow the $\mathrm{H}$-bond of the carbonyl with the catalysts (Figure 8d). 

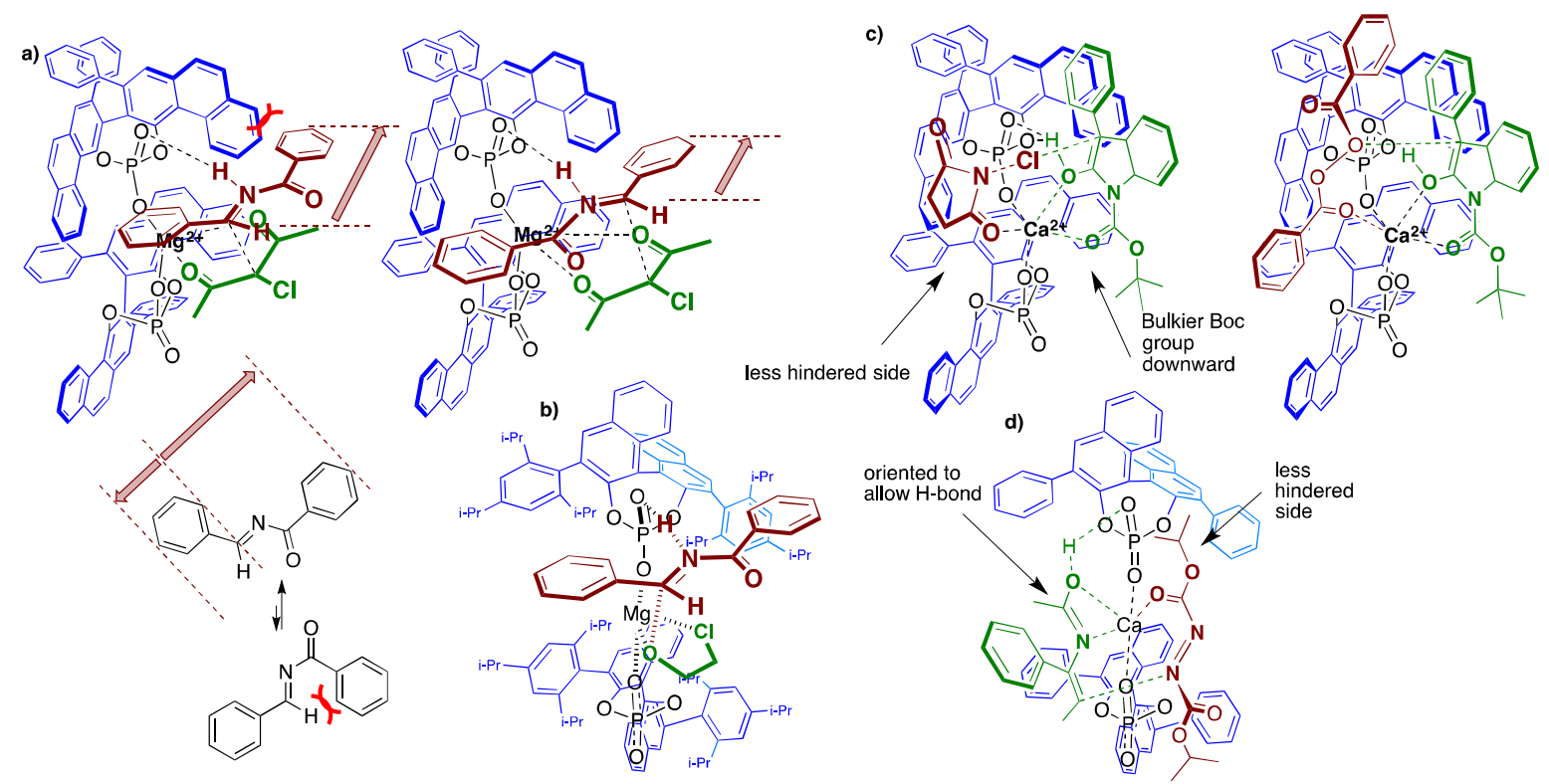

Figure 8. Model for reactions catalysed by (VAPOL) $)_{2} \mathrm{Mg}^{2+},(\mathrm{VAPOL})_{2} \mathrm{Ca}^{2+}$, and $(\mathrm{BINOP})_{2} \mathrm{Ca}^{2+}$ catalysts.

Reaction catalysed by $\mathbf{4 a} \cdot \mathbf{H}$ and $\mathbf{4 b} \cdot \mathbf{H}$ catalysts. Computational studies indicate ${ }^{39-51}$ that CPA catalysts are bifunctional and activate electrophile and nucleophile simultaneously by $\mathrm{H}$-bond interactions/proton-transfer. Accordingly, the imine and enol interact with a different phosphate oxygen atom. Considering all possible conformations of the reagents (collected in the SI section, not only the structures shown in figure 9), calculations predict the $(S)$ product will be formed in $57 \%$ and $84 \%$ ee with the catalysts $\mathbf{4 a} \cdot \mathbf{H}$ and $\mathbf{4 b} \cdot \mathbf{H}$, respectively (with an alternative quasi-RRHO correction these values change to $64 \%$ and $93 \%$ ee). This is the major enantiomer formed experimentally, with selectivities of $27 \%$ and $49 \%$ ee for the two catalysts. It is not possible to discount the possibility that residual traces of $\mathrm{Ca}^{2+}$ or other cation(s) reduce the experimentally determined ee. We suggest that this should be considered routinely when calculations predict higher levels of selectivity.

In these TS structures proton transfer from enol to the phosphate is relatively advanced (the $\mathrm{O}-\mathrm{H}$ bond length increases to >1.3 $\AA$ ). Accordingly, the nucleophile's conformational preference in the TS reflects that of the enolate, and in all low energy TS structures an E-s-cis conformation is adopted.

TSs with an s-cis or s-trans conformation of the $\mathrm{H}$-Bond

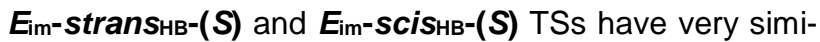
lar energies for catalysts $\mathbf{4 a} \cdot \mathbf{H}$ and $\mathbf{4 b} \cdot \mathbf{H}$, but the $s$-cis conformation of the $\mathrm{H}$-Bond is $2.0 \mathrm{kcal} / \mathrm{mol}$ more stable than the $s$-trans conformation for catalyst $\mathbf{4 h} \cdot \mathbf{H}$. This can be explained by steric interactions between acetyl group of 2 not involved in $\mathrm{H}$-bonds and the side groups of catalysts $\mathbf{4 a} \cdot \mathbf{H}$ and $\mathbf{4 b} \cdot \mathbf{H}$ in $\boldsymbol{E}_{\mathrm{im}-\boldsymbol{S C}_{\mathbf{H B}}-(S)} \mathrm{TS}$.
A frontal view of the catalysts (Figure 9) show that the upper-right and lower left quadrants are blocked for $\mathbf{4 a} \cdot \mathbf{H}$ and $\mathbf{4 b} \cdot \mathbf{H}$, but not for $\mathbf{4 h} \cdot \mathbf{H}$. Overlaying TSs from $\mathbf{4 a} \cdot \mathbf{H}$ and $\mathbf{4 b} \cdot \mathbf{H}$ with analogous $\mathbf{4 h} \cdot \mathbf{H}$ TSs reveal the rotation of the acyl-imine 1 and enol 2 reagents to prevent steric crash in these regions (Figure 9, wireframe model). Although this difference is noticeable in all TSs pairs, in the

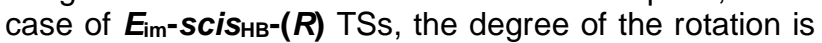
limited to an extent to which the enol $\mathbf{2}$ is still close enough to the phosphate oxygen atom to facilitate proton transfer. This limitation contributes to increase the energy of these TS structures, which, on the other side, do not suffer from the steric interactions between the enol 2 and the catalyst. The sum of the two effects yields energies only 0.2 (for $\mathbf{4 a}$ ) and 1.1 (for $\mathbf{4 b}$ ) $\mathrm{kcal} / \mathrm{mol}$ larger

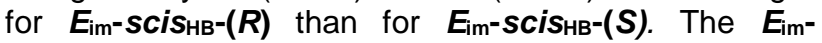
strans $_{\mathrm{HB}}-(\boldsymbol{R})$ TS structures are also less stable $(2.2$ $\mathrm{kcal} / \mathrm{mol}$ for $\mathbf{4 a}$ and $1.5 \mathrm{kcal} / \mathrm{mol}$ for $\mathbf{4 b}$ ) but in a similar extent than for $\mathbf{4 h}$ catalyst $(2.3 \mathrm{kcal} / \mathrm{mol})$, indicating that steric effects does not affect these structures.

Steric contacts involving the $t$-Bu group and the enol lead to higher energies in TSs including a $Z$-imine. The

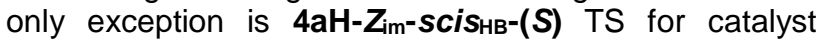
$\mathbf{4 a} \cdot \mathbf{H}$, which has a $0.7 \mathrm{kcal} / \mathrm{mol}$ higher energy than its $E$ imine analogue TS (for catalysts $\mathbf{4 b} \cdot \mathbf{H}$ or $\mathbf{4 h \cdot H}$ the energy differences are larger: 2.7 and $3.3 \mathrm{kcal} / \mathrm{mol}$ higher). A possible explanation is that the more compact $Z$ acylimine prevents steric interactions with the side substituents placed in the lower-right quadrant in a frontal representation. 


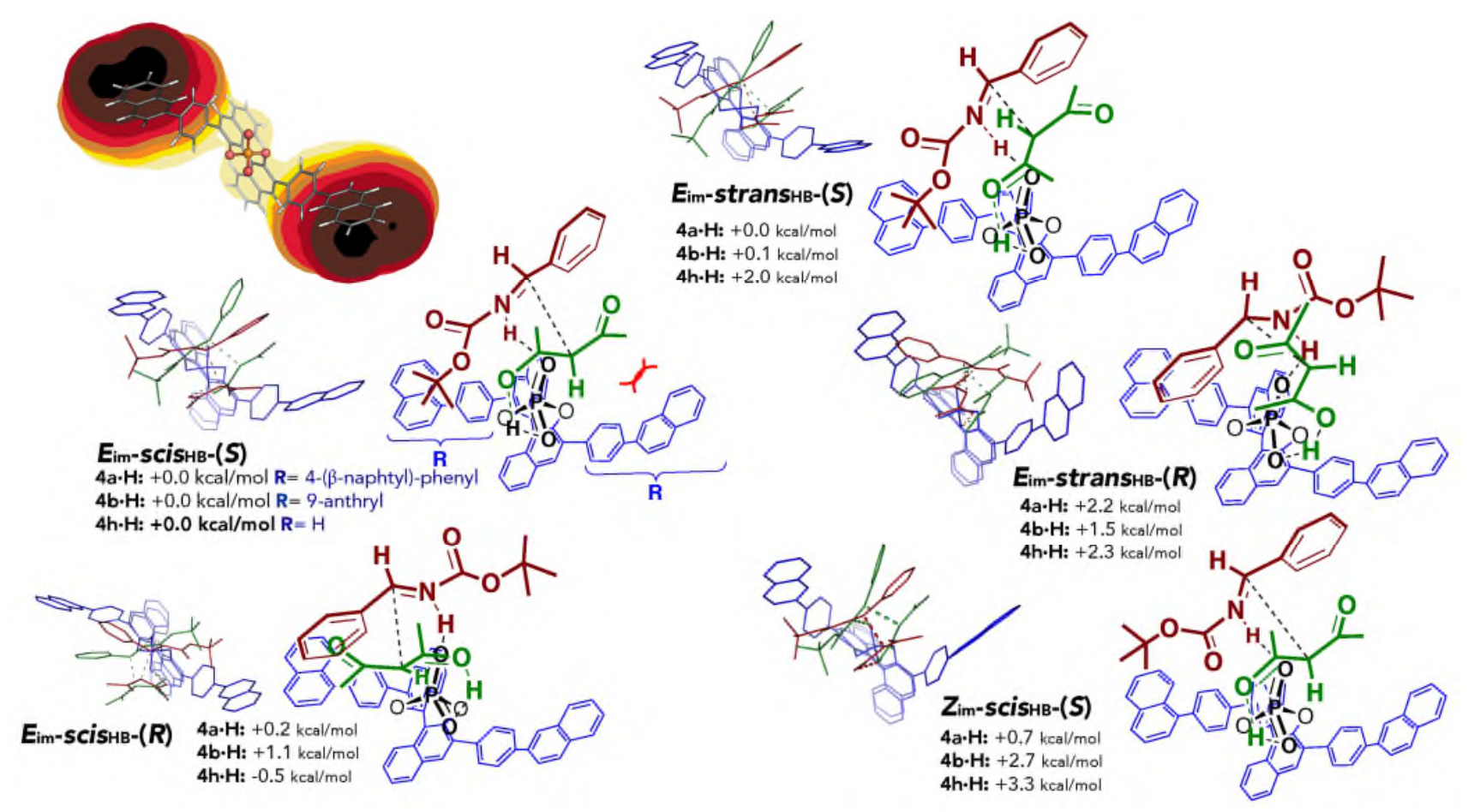

Figure 9. a) Steric map for catalyst $\mathbf{4 a \cdot H}$; b) TSs found for the reaction catalysed by $\mathbf{4 a} \cdot \mathbf{H}$. left: overlay of $\mathbf{4 a} \cdot \mathbf{H}$ and $\mathbf{4 h} \cdot \mathbf{H}$ structures. Right: schematic representation. 3-D representations of these structures are in the Supporting Information.

From these calculations, it is possible to account for the poor chiral discrimination of $\mathbf{4 a \cdot H}$ and $\mathbf{4 b} \cdot \mathbf{H}$ catalysts and why the sense of enantioselectivity is reversed using $\mathrm{Ca}$ (II) and $\mathrm{Mg}$ (II) CPA salts. The steric map of the protic catalyst (Figure $\mathbf{8 a}$ ) is similar to that of $[4 \mathbf{a}]_{2} \mathbf{M g}$, and in both cases the steric effects can be reduced placing the largest groups in the top-right or bottom-left quadrants. However, in TS structures for $[4 a]_{2} \mathrm{Mg}$ or $[4 \mathrm{a}]_{2} \mathrm{Ca}$ the enolate is fixed horizontally (mechanism Ilb) or vertically (for catalyst Ila), which also forces the imine to be oriented accordingly. For $\mathbf{4 a} \cdot \mathbf{H}$ and $\mathbf{4 b} \cdot \mathbf{H}$ the enolate is bound to the catalyst by a single $\mathrm{H}$-bond; this, along with the small size of $\mathbf{2}$, allows rotating the reagents, that are arranged diagonally in the catalyst cavity, neither incurring steric interactions with 2 nor weakening its interactions with the phosphate. The stability of the TS structures is not directly conditioned by the steric effects with the imine 1, but for the possibility of reducing these steric interactions by rotating the reagents without affecting to the interactions with the phosphates. Rotation is more difficult for more sterically demanding nucleophiles, leading to higher enantioselectivity as seen experimentally in the reactions of bulkier 1,3 dicarbonyl compounds catalysed by $\mathbf{4 b} \cdot \mathbf{H}^{73}$ Direct extrapolation from our results, however, is difficult, since in these other nucleophiles the E-s-cis conformation might not be privileged (or even possible to achieve).

\section{CONCLUSION}

The mechanism of the reaction between the N-Boc imine of benzaldehyde 1 and acetyl acetone 2, catalysed by CPA catalysts $\mathbf{4 a}$ and $\mathbf{4 b}$, and by the $\mathrm{Ca}^{2+}$ and $\mathrm{Mg}^{2+}$ salts of $\mathbf{4 a}$, has been investigated by ONIOM QM:MM methods.
For catalysts $[4 \mathbf{a}]_{2} \mathrm{Mg}$ and $[4 \mathrm{a}]_{2} \mathrm{Ca}$ we examined several mechanisms. Mechanisms involving ligand exchange between a CPA ligand and enolate are predicted to be unimportant. Despite favourable thermodynamics for ligand exchange, activation barriers were more than 20 $\mathrm{kcal} / \mathrm{mol}$ higher than more favorable pathways. Catalysts which retain both CPA ligands give much lower barriers, and selectivities consistent with experiment. Two competitive mechanisms have emerged from our work: in mechanism Ila', one of the enol oxygen atoms is $\mathrm{H}$ bonded to the catalyst, while the central metal atom is coordinated to the enol oxygens, nitrogen and oxygen $\mathrm{N}$ Boc atoms, and to the CPA phosphates. This is the most highly enantioselective pathway and is favored by $\mathrm{Ca}^{2+}$ phosphates. In mechanism Ilb, the imine accepts an $\mathrm{H}$ bond from the catalyst, and the metal center binds to CPA phosphate and enolate oxygen atoms. This is favoured by $\mathrm{Mg}^{2+}$ phosphates.

More rigid fixation (as compared to $\mathbf{4 a} \cdot \mathbf{H}$ and $\mathbf{4 b} \cdot \mathbf{H}$ catalysts) of the enolate allows a better discrimination of the diastereomeric TSs by these catalysts. The enolate is in the Z-s-cis conformation, allowing for simultaneous coordination and/or $\mathrm{H}$-bonding with the catalyst. The enolate is anchored to at least two points to the catalyst and cannot be reoriented to accommodate the acyl-imine in an orientation that prevents steric clashes with the catalyst. However, for $\mathrm{Mg}^{2+}$ catalyst and mechanism Ilb, this steric hindrance is reduced by adopting a $Z$ acyl-imine conformation. In the case of mechanism Ila' and $\mathrm{Ca}^{2+}$, steric effects with the catalyst side substituents determine the preferred placement and orientation of the acylimine (that is fixed in the $Z$ imine conformation to allow interactions between the $\mathrm{Ca}^{2+}$ atom and the acyl-imine carbonyl Oxygen) leading to higher levels of asymmetric induction. 
The proton relay mechanism occurs with catalysts $\mathbf{4 a} \cdot \mathbf{H}$ and $\mathbf{4 b} \cdot \mathbf{H}$. This gives low enantioselectivities. The enol of 2 can adopt multiple conformations, although $E$-s-cis is predominant in the TSs. This can be ascribed to the higher stability of this enolate conformation, considering that in these TSs the transfer of the enol proton to the CPA is advanced with respect to the C-C bond formation. The electrophile, acyl-imine 1, is larger than the nucleophile and it is oriented in the catalyst active site to avoid steric interactions with the 4-( $\beta$-naphththyl)-phenyl or 9-anthryl groups of the catalyst.

These results explain why $\mathbf{4 a} \cdot \mathbf{H}$ and $[4 \mathbf{a}]_{2} \mathrm{Ca}$ catalysts produce opposite enantiomers. Experimentally, trace impurities of $[\mathbf{4 a}]_{2} \mathbf{C a}$ in $\mathbf{4 a} \cdot \mathbf{H}$ can invert the sense of enantioselectivity compared to the result of using the purified protic catalyst, towards a product configuration consistent with the calcium phosphate's innate selectivity. $[4 \mathbf{a}]_{2} \mathbf{C a}$ is more enantioselective catalyst than $\mathbf{4 a} \cdot \mathbf{H}$, but it is presumably also present in much smaller amounts, indicative that it is also a more reactive catalyst than the protic form. This hypothesis requires that the energy barrier for the reaction catalysed by $[4 \mathbf{a}]_{2} \mathrm{Ca}$ is smaller than for the reaction catalysed by $\mathbf{4 a} \cdot \mathbf{H}$, and the difference should be large enough so the reactivity difference can compensate for the smaller amount of the $\mathrm{Ca}$ (II) salt. From the TSs shown, it is possible to calculate the difference on the Gibbs free energy barrier for the $[4 \mathbf{a}]_{2} \mathbf{C a}$ and $\mathbf{4 a} \cdot \mathbf{H}$ catalyst: $19.4-21.0 \mathrm{kcal} / \mathrm{mol}$ in favour of the $\mathrm{Ca}$ (II) salt.

\section{Supporting Information}

Computational methods. Alternative mechanisms for the reactions catalysed by $[4 \mathrm{a}] 2 \mathrm{Mg}$ and $[4 \mathrm{a}] 2 \mathrm{Ca}$. Different orientations of $\mathbf{4 a}$ in the TSs corresponding to Ila, Ila' and IIb mechanisms. Alternatives to the reactive conformations for mechanism la and Ib. Distortion/Interaction analysis of the TSs for $[4 \mathbf{a}]_{2} \mathbf{C a}$ and $\left[\mathbf{4 a}_{2} \mathbf{M g}\right.$ catalysts. TS structures for the proton transfer after the addition step catalyzed by $[4 \mathrm{a}]_{2} \mathrm{Ca}$ catalyst for mechanism lla'. Cartesian coordinates and absolute energies of all structures.

\section{AUTHOR INFORMATION}

\section{Corresponding Author}

*Isimon@usal.es; * robert.paton@chem.ox.ac.uk

\section{Author Contributions}

The manuscript was written through contributions of both authors. Both authors have given approval to the final version of the manuscript. Both authors contributed equally.

\section{Funding Sources}

We thank the University of Salamanca for financial support (18.K114).

\section{ACKNOWLEDGMENT}

This article is dedicated to the memory of Prof. Keiji Morokuma. whose pioneering contributions have greatly enriched the chemical sciences. We acknowledge the employment of University of Salamanca server housing service. We thank A. Lopéz García and J. A. Gonzaléz Ramos for IT support. We acknowledge the Dirac cluster at Oxford (EP/L015722/1) and the Engineering Discovery Environment (XSEDE) Comet cluster at SDSC and Bridges cluster at PSC through allocation TG-CHE180006.

\section{ABBREVIATIONS}

CPA: Chiral Phosphoric Acid. TS: Transition State.

\section{REFERENCES}

(1) Zamfir, A.; Schenker, S.; Freund, M.; Tsogoeva, S. B. Org Biomol. Chem. 2010, 8, 5262-5276.

(2) Rueping, M.; Kuenkel, A.; Atodiresei, I. Chem. Soc. Rev. 2011, 40, 4539-4549.

(3) Schenker, S.; Zamfir, A.; Freund, M.; Tsogoeva, S. B. Eur. J. Org. Chem. 2011, 2011, 2209-2222.

(4) Parmar, D.; Sugiono, E.; Raja, S.; Rueping, M. Chem. Rev. 2014, 114, 9047-9153.

(5) Terada, M. Chem. Commun. 2008, 2008, 4097-4112.

(6) Adair, G.; Mukherjee, S.; List, B. Aldrichimica Acta 2008, 41, 31-39.

(7) Rueping, M.; Koenigs, R. M.; Atodiresei, I. Chem.--Eur. J. 2010, 16, 9350-9365.

(8) Yu, J.; Shi, F.; Gong, L.-Z. Acc. Chem. Res. 2011, 44, 11561171.

(9) Akiyama, T. Chem. Rev. 2007, 107, 5744-5758.

(10) You, S.-L. Chem.--Asian J. 2007, 2, 820-827.

(11) Rueping, M.; Antonchick, A. P.; Brinkmann, C. Angew. Chem. Int. Ed. 2007, 46, 6903-6906.

(12) Wang, Y.; Zheng, K.; Hong, R. J. Am. Chem. Soc. 2012, 134, 4096-4099.

(13) Chen, W.; Hartwig, J. F. J. Am. Chem. Soc. 2013, 135, 2068-2071

(14) Terada, M.; Li, F.; Toda, Y. Angew. Chem. Int. Ed. 2013, 53, 235-239.

(15) Liu, B.; Liu, T.-Y.; Luo, S.-W.; Gong, L.-Z. Org. Lett. 2014, 16, 6164-6167.

(16) Ren, Y.-Y.; Wang, Y.-Q.; Liu, S. J. Org. Chem. 2014, 79, $11759-11767$.

(17) Shi, S.-H.; Huang, F.-P.; Zhu, P.; Dong, Z.-W.; Hui, X.-P. Org. Lett. 2012, 14, 2010-2013.

(18) Chen, J.; Wen, X.; Wang, Y.; Du, F.; Cai, L.; Peng, Y. Org Lett 2016, 18, 4336-4339.

(19) Ingle, G. K.; Liang, Y.; Mormino, M. G.; Li, G.; Fronczek, F. R.; Antilla, J. C. Org. Lett. 2011, 13, 2054-2057.

(20) Larson, S. E.; Li, G.; Rowland, G. B.; Junge, D.; Huang, R.; Woodcock, H. L.; Antilla, J. C. Org. Lett. 2011, 13, 21882191.

(21) Chen, L.; Zhang, L.; Lv, J.; Cheng, J.-P.; Luo, S. Chem.-Eur. J. 2012, 18, 8891-8895.

(22) Nimmagadda, S. K.; Zhang, Z.; Antilla, J. C. Org. Lett. 2014, 16, 4098-4101.

(23) Li, G.; Liang, T.; Wojtas, L.; Antilla, J. C. Angew. Chem. Int. Ed. 2013, 52, 4628-4632.

(24) Lalli, C.; Dumoulin, A.; Lebée, C.; Drouet, F.; Guérineau, V.; Touboul, D.; Gandon, V.; Zhu, J.; Masson, G. Chem.--Eur. J. 2014, 21, 1704-1712.

(25) Zheng, W.; Zhang, Z.; Kaplan, M. J.; Antilla, J. C. J. Am. Chem. Soc. 2011, 133, 3339-3341.

(26) Liang, T.; Li, G.; Wojtas, L.; Antilla, J. C. Chem. Commun. 2014, 50, 14187-14190.

(27) Li, X. Y.; Yuan, W. Q.; Tang, S.; Huang, Y. W.; Xue, J. H.; Fu, L. N.; Guo, Q. X. Org Lett 2017, 19, 1120-1123.

(28) Mao, Z.; Li, W.; Shi, Y.; Mao, H.; Lin, A.; Zhu, C.; Cheng, Y. Chem.--Eur. J. 2013, 19, 9754-9759.

(29) Drouet, F.; Lalli, C.; Liu, H.; Masson, G.; Zhu, J. Org. Lett. 2010, 13, 94-97.

(30) Zhang, Z.; Zheng, W.; Antilla, J. C. Angew. Chem. Int. Ed. 2010, 50, 1135-1138.

(31) Wang, C.; Han, Z.-Y.; Luo, H.-W.; Gong, L.-Z. Org. Lett. 2010, 12, 2266-2269. 
(32) Liao, S.; List, B. Angew. Chem. Int. Ed. 2010, 49, 628-631. (33) Yang, L.; Zhu, Q.; Guo, S.; Qian, B.; Xia, C.; Huang, H. Chem.--Eur. J. 2010, 16, 1638-1645.

(34) Lin, J. S.; Wang, F. L.; Dong, X. Y.; He, W. W.; Yuan, Y.; Chen, S.; Liu, X. Y. Nat Commun 2017, 8, 14841.

(35) Lv, J.; Luo, S. Chem. Commun. 2013, 49, 847-858.

(36) Barbazanges, M.; Augé, M.; Moussa, J.; Amouri, H.; Aubert, C.; Desmarets, C.; Fensterbank, L.; Gandon, V.; Malacria, M.; Ollivier, C. Chem.--Eur. J. 2011, 17, 13789-13794.

(37) Inamdar, S. M.; Konala, A.; Patil, N. T. Chem. Commun. 2014, 50, 15124-15135.

(38) Lv, J.; Zhang, L.; Hu, S.; Cheng, J.-P.; Luo, S. Chem.--Eur. J. 2011, 18, 799-803.

(39) Simón, L.; Goodman, J. M. J. Am. Chem. Soc. 2008, 130, 8741-8747.

(40) Marcelli, T.; Hammar, P.; Himo, F. Chem.--Eur. J. 2008, $14,8562-8571$.

(41) Marcelli, T.; Hammar, P.; Himo, F. Adv. Synth. Catal. 2009, 351, 525-529.

(42) Simón, L.; Goodman, J. M. J. Am. Chem. Soc. 2009, 131, 4070-4077.

(43) Shi, F.-Q.; Song, B.-A. Org. Biomol. Chem. 2009, 7, 12921298.

(44) Akiyama, T.; Morita, H.; Bachu, P.; Mori, K.; Yamanaka, M.; Hirata, T. Tetrahedron 2009, 65, 4950-4956.

(45) Li, N.; Chen, X.-H.; Song, J.; Luo, S.-W.; Fan, W.; Gong, L.-Z. J. Am. Chem. Soc. 2009, 131, 15301-15310.

(46) Simón, L.; Goodman, J. M. J. Org. Chem. 2010, 75, 589597.

(47) Zheng, C.; Sheng, Y.-F.; Li, Y.-X.; You, S.-L. Tetrahedron 2010, 66, 2875-2880.

(48) Hong, X.; Küçük, H. B.; Maji, M. S.; Yang, Y.-F.; Rueping, M.; Houk, K. N. J. Am. Chem. Soc. 2014, 136, 13769-13780.

(49) Jindal, G.; Sunoj, R. B. Angew. Chem. Int. Ed. 2014, 53, $4432-4436$.

(50) Seguin, T. J.; Wheeler, S. E. ACS Catal. 2016, 2681-2688. (51) Sunoj, R. B. Acc. Chem. Res. 2016, 49, 1019-1028.

(52) Simón, L. Org. Biomol. Chem. 2018, 10.1039/C1037OB02875J.

(53) Xu, S.; Wang, Z.; Li, Y.; Zhang, X.; Wang, H.; Ding, K. Chem.--Eur. J. 2010, 16, 3021-3035.

(54) Terada, M.; Komuro, T.; Toda, Y.; Korenaga, T. J. Am. Chem. Soc. 2014, 136, 7044-7057.

(55) Monaco, M. R.; Fazzi, D.; Tsuji, N.; Leutzsch, M.; Liao, S.; Thiel, W.; List, B. J Am Chem Soc 2016, 138, 14740-14749.

(56) Renzi, P.; Hioe, J.; Gschwind, R. M. J Am Chem Soc 2017, 139, 6752-6760.

(57) Simón, L.; Goodman, J. M. J. Org. Chem. 2011, 76, 17751788.

(58) Reid, J. P.; Simón, L.; Goodman, J. M. Acc Chem Res 2016, 49, 1029-1041.

(59) Changotra, A.; Das, S.; Sunoj, R. B. Org Lett 2017, 19, 2354-2357.

(60) Reid, J. P.; Goodman, J. M. J Am Chem Soc 2016, 138, 7910-7917.

(61) Reid, J. P.; Goodman, J. M. Chem.-- Eur. J. 2017, 23, 14248-14260.

(62) Jindal, G.; Sunoj, R. B. J. Org. Chem. 2014, 79, 76007606.

(63) Jindal, G.; Sunoj, R. B. J. Am. Chem. Soc. 2014, 136, 15998-16008.

(64) Jindal, G.; Sunoj, R. B. Org. Lett. 2015, 17, 2874-2877.

(65) Avila, C. M.; Patel, J. S.; Reddi, Y.; Saito, M.; Nelson, H. M.; Shunatona, H. P.; Sigman, M. S.; Sunoj, R. B.; Toste, F. D. Angew Chem Int Ed 2017, 56, 5806-5811.

(66) Orlandi, M.; Hilton, M. J.; Yamamoto, E.; Toste, F. D.; Sigman, M. S. J Am Chem Soc 2017, 139, 12688-12695.
(67) Zhu, Y.; He, W.; Wang, W.; Pitsch, C. E.; Wang, X.; Wang, $X$. Angew Chem Int Ed 2017, 56, 12206-12209.

(68) Jindal, G.; Kisan, H. K.; Sunoj, R. B. ACS Catal. 2014, 480-503.

(69) Kisan, H. K.; Sunoj, R. B. J. Org. Chem. 2015, 80, 21922197.

(70) Hopmann, K. H. Chem.--Eur. J. 2015, 21, 10020-10030.

(71) Grayson, M. N.; Krische, M. J.; Houk, K. N. J Am Chem Soc 2015, 137, 8838-8850.

(72) Duarte, F.; Paton, R. S. J Am Chem Soc 2017, 139, 88868896.

(73) Hatano, M.; Moriyama, K.; Maki, T.; Ishihara, K. Angew. Chem. Int. Ed. 2010, 49, 3823-3826.

(74) Jiang, G.; List, B. Angew. Chem. Int. Ed. 2011, 50, 94719474.

(75) Johnston, C. P.; Kothari, A.; Sergeieva, T.; Okovytyy, S. I.; Jackson, K. E.; Paton, R. S.; Smith, M. D. Nat Chem 2015, 7, 171-177.

(76) Guan, Y.; Wheeler, S. E. Angew. Chem. Int. Ed. 2017, 56, 9101-9105

(77) Belova, N. V.; Oberhammer, H.; Trang, N. H.; Girichev, G. V. J. Org. Chem. 2014, 79, 5412-5419.

(78) Belova, N. V.; Oberhammer, H.; Girichev, G. V. J. Phys. Chem. A. 2004, 108, 3593-3597.

(79) Simón, L.; Goodman, J. M. J. Am. Chem. Soc. 2012, 134, 16869-16876.

(80) Simón, L.; Goodman, J. M. Org. Biomol. Chem. 2009, 7, 483-487.

(81) Simón, L.; Paton, R. S. J. Org. Chem. 2015, 80, 27562766.

(82) Simón, L.; Paton, R. S. Org Biomol Chem 2016, 14, 30313039.

(83) Simon, L.; Paton, R. S. J Org Chem 2017, 82, 3855-3863.

(84) Grayson, M. N.; Pellegrinet, S. C.; Goodman, J. M. J. Am. Chem. Soc. 2012, 134, 2716-2722.

(85) Funes-Adoiz, I.; R. Paton, GoodVibes v2.0.1, 2017 , https://github.com/bobbypaton/GoodVibes.

(86) Grimme, S. Chem.--Eur. J. 2012, 18, 9955-9964.

(87) Ribeiro, R. F.; Marenich, A. V.; Cramer, C. J.; Truhlar, D. G. J Phys Chem B 2011, 115, 14556-14562.

(88) Grimme, S.; Antony, J.; Ehrlich, S.; Krieg, H. J. Chem. Phys. 2010, 132, 154104.

(89) Grimme, S. Wiley Interdisciplinary Reviews: Computational Molecular Science 2011, 1, 211-228.

(90) Kruse, H.; Goerigk, L.; Grimme, S. J. Org. Chem. 2012, 77, 10824-10834.

(91) Grimme, S.; Schreiner, P. R. Angew Chem Int Ed Engl 2011, 50, 12639-12642.

(92) Wagner, J. P.; Schreiner, P. R. Angew Chem Int Ed Engl 2015, 54, 12274-12296.

(93) Peng, Q.; Duarte, F.; Paton, R. S. Chem. Soc. Rev. 2016, 45, 6093-6107.

(94) Plata, R. E.; Singleton, D. A. J. Am. Chem. Soc. 2015, 137, 3811-3826.

(95) Besora, M.; Vidossich, P.; Lledos, A.; Ujaque, G.; Maseras, F. J Phys Chem A 2018122 (5), 1392-1399.

(96) Liu, Z.; Patel, C.; Harvey, J. N.; Sunoj, R. B. Phys Chem Chem Phys 2017, 19, 30647-30657.

(97) Unlike mechanism Ilb, the formation of the product following mech- anism Ila' path requires a final proton transfer to the product. The TSs for this proton transfer have a lower energy than any of the TSs for the C-C bond formation; therefore, proton transfer does not influence enantioselec- tivity; see details in SI section.

(98) Gridnev, I. D.; Kouchi, M.; Sorimachi, K.; Terada, M. Tetrahedron Lett. 2007, 48, 497-500. 
Graphic entry for the Table of Contents (TOC:

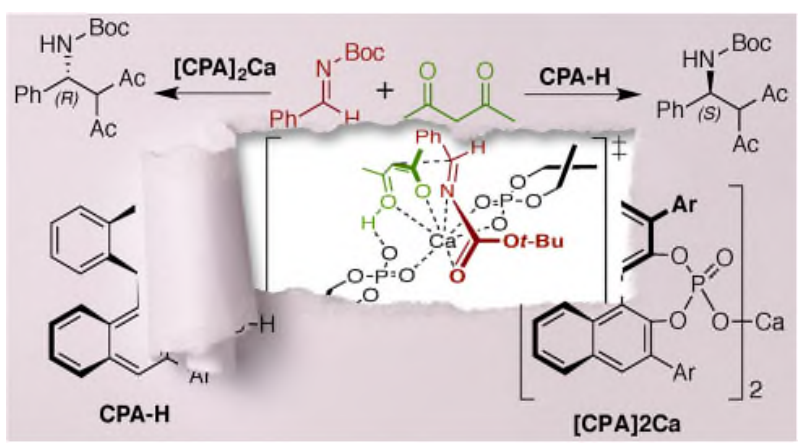

